\title{
Radiation Response of Nanocrystalline Rutile $\left(\mathrm{TiO}_{2}\right)$
}

\author{
Jiaming Zhang ${ }^{*}$, Jie Lian ${ }^{* *}$, Fereydoon Namavar ${ }^{* * *}$, R. C. Ewing ${ }^{*}$ \\ *Departments of Geological Sciences and Materials Science \& Engineering, University of Michigan, \\ Ann Arbor, Michigan 48109-1005, USA \\ ** Department of Mechanical, Aerospace \& Nuclear Engineering, Rensselaer Polytechnic Institute, \\ Troy, NY 12180 \\ ${ }^{* * *}$ University of Nebraska Medical Center, Omaha, NE 68198, USA
}

Titanium dioxide $\left(\mathrm{TiO}_{2}\right)$ has a wide range of technological applications, such as photocatalysis, gas sensors, and thin film electronic devices [1]. There are three polymorphs of $\mathrm{TiO}_{2}$ : anatase, rutile, and brookite. Ion irradiation effects in rutile phases have been extensively studied because doping with ions can modify its physical and optical properties for electrical and optical applications [2]. Previous work on irradiation effects in $\mathrm{TiO}_{2}$ polymorphs have focused on its response in bulk form [3]. In this study, we provide a systematic irradiation study of nanocrystalline, thin-films of rutile grown by ion beam-assisted deposition (IBAD). In situ TEM characterization was used to study the phase stability and microstructural evolution of nanocrystalline rutile upon energetic ion beam bombardment.

The nanocrystalline rutile thin films were prepared by IBAD at the Nanotechnology Laboratory of the University of Nebraska Medical Center [4]. IBAD combines an electron beam evaporation system with an ion beam bombardment in a high vacuum environment with a base pressure at $10^{-8}$ torr. Source material was $99.9 \%$ pure rutile from Alfa Aesar and was deposited at a rate of $0.5 \AA / \mathrm{s}$ (for amorphous region) to $2.5 \AA / \mathrm{s}$ (for nanocrystalline region). Ion beam consist of mixture of argon and oxygen with a constant current density of $200 \mu \mathrm{A} / \mathrm{cm}^{2} . \quad \mathrm{TiO}_{2}$ was deposited into silicon and glass substrates without any additional heating. Cross-sectional TEM samples were then prepared for ion irradiation and in situ TEM characterization using the IVEM-Tandem Facility at the Argonne National Laboratory. Ion irradiations were performed using 1-MeV Kr${ }^{+}$ions between 300 and 1073 K. Ex situ high resolution TEM (HRTEM) imaging was performed using a JEOL JEM 2010F electron microscope.

Fig. 1a shows a cross-sectional dark field TEM image of an as-deposited $\mathrm{TiO}_{2}$ thin film $(\sim 400 \mathrm{~nm}$ thick) on a Si wafer. There is an amorphous buffer layer (created by a high current intensity argon beam or a high ion to atom arrival ratio) between the crystalline $\mathrm{TiO}_{2}$ and $\mathrm{Si}$-substrate in order to further enhance the adhesion. The selected area electron diffraction (SAED) pattern (inset in Fig. 1d) from the near-surface shows that the nanocrystalline thin film is rutile $\left(P 4 / \mathrm{mnm}, a_{0}=0.459 \mathrm{~nm}\right.$, $c_{0}=0.296 \mathrm{~nm}$ ) and is highly textured. EDX measurements (data not shown here) show that the nanocrystalline film and amorphous buffer layer contain $\mathrm{Ti}$ and $\mathrm{O}$ without observable impurities. HRTEM image in Fig. 1d shows that the crystal grain size is 5-10 nm with significant lattice distortion, which may be caused by the high strain field with significant oxygen vacancies during the deposition process.

The phase stability of nanocrystalline rutile was investigated by energetic beam bombardment. An ion beam-induced rutile-to-amorphous phase transformation occurred upon $1 \mathrm{MeV} \mathrm{Kr}^{2+}$ irradiation at $300 \mathrm{~K}$ (as shown in the images in Fig. 1), which is consistent with observations of the bulk form 
[2]. There is no obvious change in the amorphous buffer layer, which suggests the amorphous phase is relatively stable under irradiation at $300 \mathrm{~K}$. In contrast, a radiation-induced re-crystallization in the amorphous layer was observed when the rutile thin film was irradiated at elevated temperature (data not shown here).

In conclusion, we have studied phase stability and microstructure evolution of nanocrystalline rutile thin film under ion beam bombardment. These results confirm the potential of using ion beam irradiation to manipulate the microstructure at the nanoscale and tailor the physical properties for technological applications.

References

[1] Y. Hwang et al., Nano Lett. 9: p. 410 (2009)

[2] F. Li et al., J. Nucl. Mater. 306: p. 121 (2002)

[3] M. Ishimaru et al., Appl. Phys. Lett. 77: p. 4151 (2000)

[4] F. Namavar et al., Nano Lett. 8: p. 988 (2008)

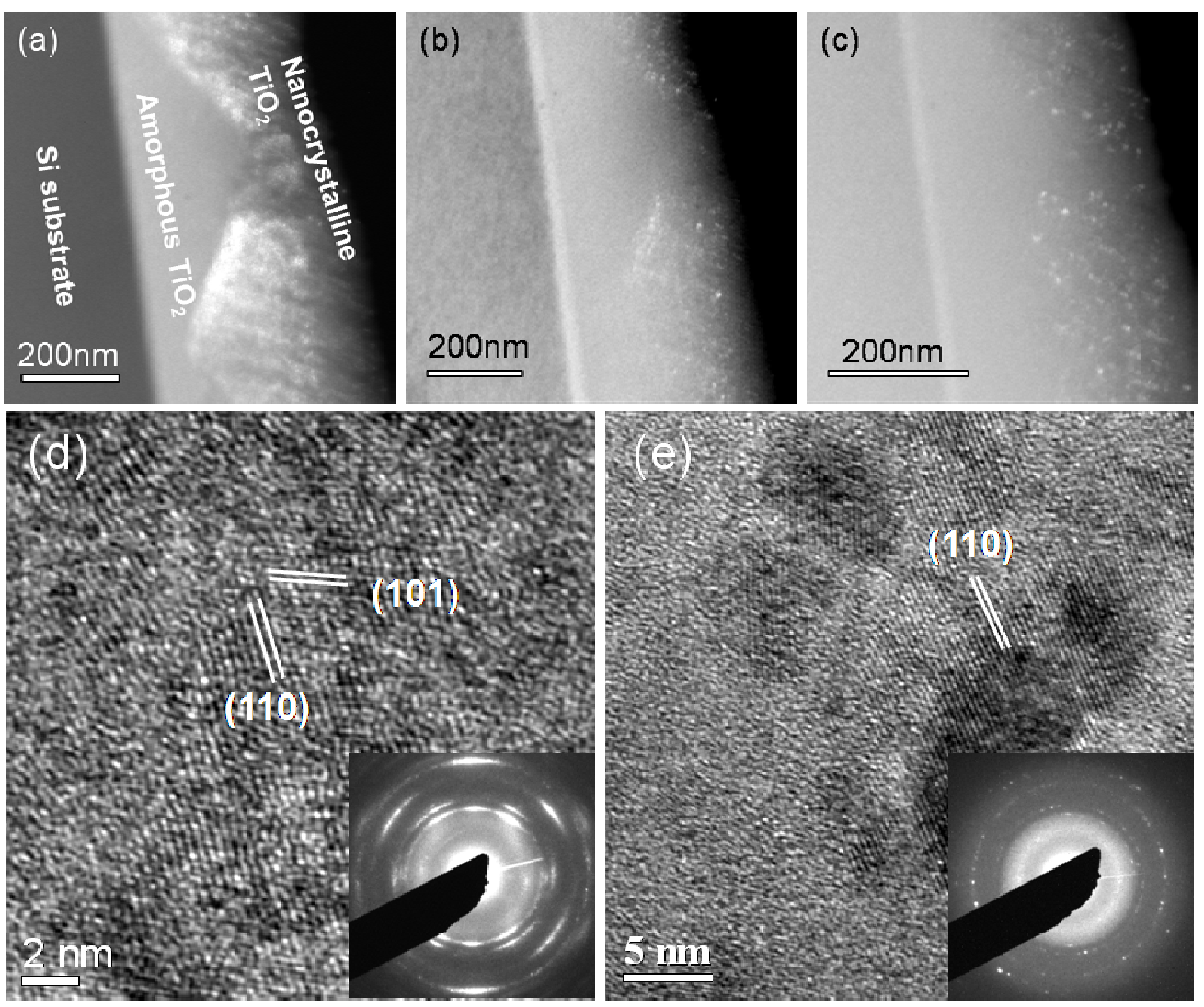

FIG. 1 Cross-sectional dark field TEM images of nanocrystalline rutile thin film on the Si-substrate upon $1 \mathrm{MeV} \mathrm{Kr}^{2+}$ irradiation at room temperature (a) as-deposited, (b) $4 \times 10^{14}$ ions $/ \mathrm{cm}^{2}$, (c) $8.75 \mathrm{x}$ $10^{14}$ ions $/ \mathrm{cm}^{2}$. HRTEM images and corresponding SAED patterns before irradiation (d) and at a fluence of $1.25 \times 10^{15}$ ions $/ \mathrm{cm}^{2}(\mathrm{e})$. 